\title{
An Ant Colony Algorithm for Time-Dependent Vehicle Routing Problem with Time Windows
}

\author{
Umman Mahir Yıldırım and Bülent Çatay
}

Sabancı University, Orhanlı, Tuzla, 34956 Istanbul, Turkey

mahiryldrm@su.sabanciuniv.edu, catay@sabanciuniv.edu

Summary. In this paper, we address the Vehicle Routing Problem with Time Windows, both time-independent and -dependent cases. In the timeindependent case, our objective is to minimize the total distance. To solve this problem, we propose an Ant Colony Optimization algorithm. Then we implement the algorithm to solve the time-dependent case where the objective is to minimize the total tour time. The time dependency is embedded in this model by using a deterministic travel speed function which is a step function of the time of the day. An experimental evaluation of the proposed approach is performed on the well-known benchmark problems.

\section{Introduction}

Optimizing a distribution network has been and remains an important challenge both in the literature and in real-life applications and the routing of a fleet of vehicles is the most widely addressed problem in a distribution network. The Vehicle Routing Problem (VRP) determines a set of vehicle routes originating and terminating at a single depot such that all customers are visited exactly once and the total demand of the customers assigned to each route does not violate the capacity of the vehicle. The objective is to minimize the total distance traveled. An implicit primary objective is to use the least number of vehicles. The Vehicle Routing Problem with Time Windows (VRPTW) is a variant of VRP in which lower and upper limits are imposed to the delivery time of each customer. The arrival at a customer outside the specified delivery time is either penalized (soft time windows) or strictly forbidden (hard time windows). The interested reader is referred to [1] for more details on VRPTW.

In the Stochastic Vehicle Routing Problem, the customer demands and/or the travel times between the customers may vary. Although 
stochastic travel times and demand distributions have been frequently used in the literature, time-varying travel speeds and time-dependent VRPTW (TDVRPTW) have seldom been addressed. In the literature, time dependency is taken into consideration in two ways: stochastic travel times and deterministic travel times. First introduced by [2], stochastic travel times are mainly examined by [4] and [3]. [5] proposed a deterministic travel time based model in which the important nonpassing property is introduced. [6] and [7] also use deterministic travel times in a setting where the day is divided into time intervals.

Many exact and heuristic solution approaches were presented for solving VRP and its extensions. One recent approach, Ant Colony Optimization $(\mathrm{ACO})$ is a population-based metaheuristic that can be used to find approximate solutions to difficult optimization problems. A detailed study of ACO and its variants can be found in [8].

In this study, an ACO approach is developed to efficiently solve VRPTW and TDVRPTW with hard time windows. In the next section, we provide a description of the two problems. In Section 3, the proposed algorithm is presented. Section 4 is devoted to the computational study and concluding remarks are given in Section 5 .

\section{Problem Description}

In VRPTW, $N$ geographically dispersed customers are serviced by a homogenous fleet of $K$ vehicles with capacity $Q$. All vehicle routes start and end at a central depot visiting each customer $i, i=1, \ldots, N$, exactly once. Each customer has a demand $q_{i}$, service time $s_{i}$ and time window $\left[e_{i}, l_{i}\right]$. The service time is the loading or unloading service times at the customer $i$ where the terms $e_{i}$ and $l_{i}$ denote the earliest and latest available service start time for customer $i$. The time window may prohibit the visit of certain customer pairs one after the other. VRPTW is in fact a special case of TDVRPTW. In TDVRPTW the travel time between any source and destination pair on the road network is not a function of the distance alone and is subject to variations due to accidents, weather conditions or other random events. Speed limitations imposed by the road type and the traffic density distribution of the road which is also affected by the time of the day are two main components that cause fluctuations in travel speeds. That is, the travel time between two customers is not constant during the entire scheduling horizon and changes with the changing sub-divisions of the horizon, called time-intervals. 
In TDVRPTW, the feasible and infeasible customer pairs are not necessarily same as in the time-independent case. A dynamic travel time calculation is required to check the feasibility in the route construction phase. The arrival time to the next customer may be realized earlier or later compared to the time-independent case.

\section{The Proposed Ant Colony Optimization Approach}

\subsection{ACO for VRPTW}

Our ACO approach is inspired from the rank-based Ant System ${ }^{1}$ introduced by [10] and is outlined as follows.

Route Construction - Initially, $N$ ants are placed at the $N$ nearest customers to the depot. After a vehicle has returned to the depot, it starts from the customer with the largest attractiveness value. To put a limit on the exploration and to speed up the algorithm, we use a candidate list which consists of the nearest $C L$ (candidate list size) neighbors of the customer. The customers are added to the list by taking their feasibility and distance into account. The next customer is selected from the candidate list using the probabilistic action choice rule as described in [10].

Local Search - In this study, two types of local search procedures, namely Swap and Move, are utilized to improve the solution quality. These procedures are applied at the end of each iteration and pheromone trails are updated afterwards. The simple idea behind the Swap procedure is to exchange two customers in a single route (intraroute) or between routes (inter-route) until no further improvements are available. The Move procedure attempts to improve the solution by removing a customer and inserting it between two other customers, intra-route or inter-route.

Pheromone Update - The pheromone levels are initialized as $N / L_{0}$, where $L_{0}$ is total distance obtained using the nearest neighbor heuristic. After all ants have constructed their tours, first the pheromone trails are evaporated at the rate $\rho$ then $k$ elitist ants are allowed to reinforce the trails. In our pheromone reinforcement strategy, we utilize $k-1$ best-ranked ants for the first $P$ iterations (referred to as preliminary iterations) and in the remainder of iterations we allow best-so-far ant along with the $k-1$ best-ranked ants to deposit pheromone. Our aim in adopting this strategy is to avoid a quick stagnation.

\footnotetext{
${ }^{1}\left(A S_{\text {rank }}\right)$
} 


\subsection{Extensions to TDVRPTW}

In TDVRPTW, the objective function and travel speeds are adapted accordingly. In addition, the local search and pheromone update procedures are modified in line with the new objective function of minimizing the total travel time. Since the scheduling horizon is divided into multiple time-intervals, the pheromone network also comprises multiple dimensions. An ant in time-interval $t$ deposits pheromone on the corresponding dimension in the network.

\section{Computational Study}

The performance of the algorithm is tested on the time-independent benchmark problems of [9] using real numbers (float precision). The time-dependent versions are obtained by dividing the scheduling horizon into three time intervals and utilizing different road types which are randomly assigned to each arc. Time-dependent travel speeds are embedded in the algorithm by utilizing a travel time matrix similar to the approach of [6]. In the preliminary runs, we observed that heuristic information such as "1/distance" or "savings" does not improve the solution quality much due to the high performance of local search. Thus, no visibility function is implemented. For each problem, 10 runs are performed with the following parameter setting: $\alpha=1, \beta=0, \rho=$ 0.15 , number of iterations $=100, P=25$, number of ants $=N, k=$ $6, C L=50$. Both one dimensional and three dimensional pheromone networks are tested in the time-independent case. The algorithm is shown to be efficient and then applied to the time-dependent case. The primary objective of the time-independent models is to minimize the total distance (TD) whereas total tour time (TT) is minimized in the time-dependent case. The algorithm is coded in $\mathrm{C \#}$ and executed on a Pentium 2.40 $\mathrm{GHz}$ processor.

Table 1 gives the average distance of each problem type for 10 runs as well as the average number of vehicles (VN). In the class column, $\mathrm{C}$ and $\mathrm{R}$ refer to problem types where the customers are clustered and uniformly randomly distributed, respectively. $\mathrm{RC}$ refers to the problem type which includes a combination of clustered and randomly distributed customers. Since a three-dimensional network is needed for time-dependent case we also experimented the effect of such network structure on the time-independent problem. For C problem sets, both one- and three-dimensional pheromone networks exhibit the same performance as a result of the clustered network structure which narrows the feasible region. One-dimensional network outperforms the 
Table 1. Average Results

\begin{tabular}{lrrrrrrrrr}
\hline & \multicolumn{4}{c}{ Time-independent } & \multicolumn{3}{c}{ Time-dependent } \\
class & TD & NV & TD & NV & TD & NV & TD & NV & TT \\
\hline C1 & 828.380 & 10.00 & 828.380 & 10.00 & 828.380 & 10.00 & 1093.140 & 10.47 & 9945.998 \\
C2 & 590.259 & 3.00 & 589.859 & 3.00 & 589.859 & 3.00 & 941.016 & 4.16 & 9854.871 \\
R1 & 1191.432 & 13.73 & 1187.282 & 13.58 & 1181.453 & 13.08 & 1499.805 & 12.72 & 2298.308 \\
R2 & 913.806 & 5.78 & 901.507 & 5.55 & 898.067 & 5.55 & 1627.551 & 3.69 & 2352.808 \\
RC1 & 1368.344 & 13.54 & 1357.403 & 13.25 & 1339.235 & 12.75 & 1645.410 & 12.64 & 2405.311 \\
RC2 & 1044.907 & 6.55 & 1027.401 & 6.50 & 1015.738 & 6.38 & 1988.114 & 4.25 & 2672.617 \\
\hline
\end{tabular}

three-dimensional network in type 1 problems where time windows are narrower and the vehicle capacities are smaller whereas a threedimensional network is more suitable for type 2 problems. Overall, we observed that a three-dimensional network slightly outperforms the one-dimensional network on the overall average solution quality. Therefore, we report the average and best results belonging to the threedimensional network setting. For comparison, we also provide bestknown solutions as reported in [11]. As seen in Table 1, our distances are comparable to the best-known distances and the average gap is only $0.66 \%$. Furthermore, we note that we have been able to improve the best-known distance of six instances.

For the time-dependent problems, we report the average tour times as well as the corresponding average distances. The distances are 48.1 $\%$ longer on the average compared to the time-independent case. This is an expected result since the two problems have different objective functions. On the other hand, the average number of vehicles is $8.89 \%$ less in time-dependent case. We also observe that type 2 problems are more sensitive to the time-dependent travel times. The distances for type 2 problems increase dramatically due to the existence of tighter constraints.

\section{Conclusion}

In this paper, we propose an ACO algorithm for solving the VRPTW and TDVRPTW. Our preliminary experimental results show that the proposed algorithm provides good quality results; however, the computation times are rather long. We have observed that the local search procedure enhances the solution quality of ACO significantly. On the other hand, a large portion of the computational time is consumed by 
the local search procedure. Further research may focus on a selective local search policy to reduce the computational effort. To improve the performance of the algorithm, a visibility function using the time window information can be implemented and a more detailed analysis on the trade-off between the solution quality and computational effort may be conducted.

\section{References}

1. Cordeau J, Desaulniers G, Desrosiers J, Solomon MM, Soumis F (2001) VRP with Time Windows. In: Toth $\mathrm{P}$ and Vigo D (Eds) The vehicle routing problem. SIAM Monographs on Discrete Mathematics and Applications. SIAM Publishing, Philadelphia, PA, pp. 157-193

2. Laporte G, Louveaux F, Mercure H (1992) The vehicle routing problem with stochastic travel times. Transportation Science 26(3):161-170

3. Potvin J, Xu Y, Benyahia I (2006) Vehicle routing and scheduling with dynamic travel times. Computers \& Operations Research 33:1129-1137

4. Kenyon AS, Morton DP (2003) Stochastic vehicle routing with random travel times. Transportation Science 37(1):69-82

5. Ahn BH, Shin JY (1991) Vehicle-routeing with time windows and timevarying congestion. Journal of Operations Research Society 42(5):393-400

6. Ichoua S, Gendreau M, Potvin JY (2003) Vehicle dispatching with time-dependent travel times. European Journal of Operations Research 144:379-396

7. Donati AV, Montemanni R, Casagrande N, Rizzoli AE, Gambardella LM (2008) Time-dependent vehicle routing problem with a multi ant colony system. European Journal of Operational Research 185:1174-1191

8. Dorigo B, Stützle T (2004) Ant colony optimization. MIT Press, Cambridge Massachusetts

9. Solomon MM (1987) Algorithms for the vehicle routing and scheduling problems with time window constraints. Operations Research 35(2):254265

10. Bullnheimer B, Hartl RF, Strauss C (1999) An Improved Ant System Algorithm for the Vehicle Routing Problem. Annals of Operations Research 89:319-328

11. Alvarenga GB, Mateus GR, Tomi G (2007) A genetic and set partitioning two-phase approach for the vehicle routing problem with time windows. Computers \& Operations Research 34:1561-1584 